\title{
Future and development direction (21-35) of M\&S for building up to the hyper-army in Republic of Korea Army:
}

Focusing on fostering high-tech armed forces

\author{
Kyoungchan Won $^{*} \cdot$ Yong-Pil Kim ${ }^{* *} \cdot$ Minsuk Kim ${ }^{* *} \cdot$ \\ Taegyun $\mathrm{Ha}^{* *} \cdot$ Yong-Yeon Lee ${ }^{* *} \cdot$ Hojun Lee ${ }^{* * *}$
}

\footnotetext{
This study presents the direction of modeling and simulation (M\&S) development, which is essential for high-tech armed forces, focusing on the Vision 2030 in Republic of Korea Army. In particular, in preparation for the Fourth Industrial Revolution, the army is doing its best to transform itself with cutting-edge science and technology. It is preparing for future warfare by promoting artificial intelligence (Al), a dronebot combat system, the warrior platform, and Army-Tiger 4.0 to

(a) (1) This work is licensed under a Creative Commons Attribution 4.0 International License.

* (First Author) Center for Army Analysis \& Simulation, M\&S Force Programming Officer, popdin@naver.com

** (Co-Author) Center for Army Analysis \& Simulation, Director of CAAS, anwlwow@naver.com; M\&S Policy Development Officer, ppippo8010@gmail.com; M\&S Based System Officer, positiveha@naver.com; M\&S Force Planning Officer, c15837@naver.com

*** (Corresponding Author) Center for Army Analysis \& Simulation, Chief of M\&S Development Department, jikimy72@naver.com
} 
increase the mobility, intelligence, and networking of infantry units. M\&S is vital for accomplishing this advanced science and technology. In other words, it is an essential element of effective defense management, such as improving defense capabilities, enhancing military power, strengthening combat readiness posture, and training for future science. In particular, M\&S is the best solution to overcome the difficult conditions of a limited budget and lack of resources by using more creative thinking methods along with cutting-edge technologies, such as Al, big data, virtual reality, and the Internet of things (IoT). The Republic of Korea Army recognizes its importance and intends to introduce and implement the 21-35 Army M\&S development direction to present the vision and direction of the Army M\&S for advanced science and technology force and to develop it continuously.

Keywords : modeling and simulation, high-tech military forces, vision 2030 in Republic of Korea Army, LVCG (Live, Virtual, Constructive, Game) training 
Future and development ... / Kyoungchan Won • Yong-Pil Kim • Minsuk Kim • Taegyun Ha • Yong-Yeon Lee • Hojun Lee 39

\section{I. 서론}

$\mathrm{M} \& \mathrm{~S}$ 는 국방 분야에서 활용되는 모델링과 시뮬레이션의 합성어로 Figure 1 과 같이 전장과 유사 한 특성과 기능을 모형화(Modeling)한 환경에서 시간의 흐름에 따라 진행하는 모의(Simulation)를 통해 실험결과를 예측 및 검증하는 과학적 기법이다. M\&S는 소요제기 결정, 획득관리, 분석평가, 교육훈련 등의 업무를 지원하는 도구이자 수단이며, 실제 실행이나 체험이 제한되는 현상에 대한 분석 또는 훈련을 모의로 해결함으로써 다양한 육군의 현안에 대한 의사결정과 실전적인 연습 - 훈 련을 지원하여 투입비용 대비 높은 효과를 낼 수 있는 핵심도구이다.



Real World

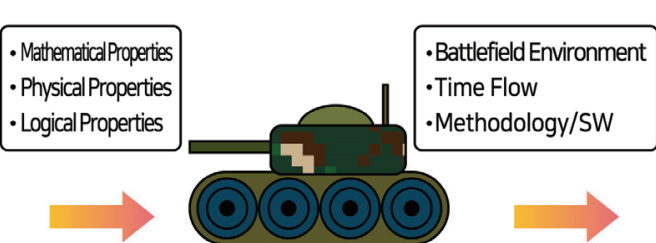

Modeling

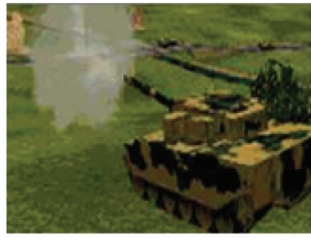

Simulation

$<$ Figure 1> Defense modeling \& simulation

$\mathrm{M} \& S$ 의 활용 분야는 연습 - 훈련, 분석, 획득, 실험 분야로 구분된다. 연습 - 훈련은 개별 병사와 승무원의 장비 조직 및 숙달 훈련으로부터 지휘관과 참모의 지휘·결심 및 절차훈련 등에 적용된 다. 분석은 전략, 전술 및 군수관리 분야의 결심 지원과 작전계획 수립, 전력소요, 부대구조에 대한 검증 및 분석평가 업무에 적용된다. 획득은 소요검증, 연구개발, 시험평가 등에 적용된다. 실험은 합동개념 및 교리 검증, 작전계획 검증, 군 구조 및 편성 검증, 무기·장비·물자의 소요검증에 적 용된다.

육군은 첨단과학기술군으로 거듭나기 위해 노력하고 있다. $\mathrm{AI}$ 와 드론봇 전투체계, 워리어플랫폼, 그리고 보병부대의 기동화 - 지능화 - 네트워크화를 추구하는 'Army-TIGER 4.0' 등을 추진하며 미 래전을 준비하고 있다(Kim, Park, Cha, \& Kim, 2021; Shin, Choi, \& Park, 2021). 미래 첨단과학기 술군으로의 도약적 변혁을 위해 육군 $\mathrm{M \& S}$ 의 비전과 방향을 설계하고, 지속적으로 발전시키고자 육 군 M\&S 발전방향을 연구하였다. 본 연구는 2019년에 발간한 육군 기본정책서인 육군 비전 20301) 을 구현하기 위해 육군 M\&S의 명확한 청사진을 정밀하게 그린 후, 이에 따른 정책추진 방향을 제 시하는 것에 의미가 있다. M\&S 발전을 위한 관련 연구는 2008년 '국방 M\&S 체계 발전방향'과 2012년에 ‘전시작전권 전환 관련 미래 육군 M\&S 체계 발전방향’이 있다. 본 연구는 기존 연구에서 제시한 M\&S의 일부인 과학화훈련체계 발전방향 외에 M\&S의 정책 및 제도, 인력 및 조직, 기술 
및 데이터 등을 포함하여 발전방향을 제시하였다. 이를 위해 $\mathrm{ADD}, \mathrm{KIDA}$, 국방부, 합참, 연합사, 육 군 참모부서 등 관련 부서의 전문가 의견을 수렴하여 기존 우리가 제시한 11 개 과제 외에 미래 $\mathrm{M} \& \mathrm{~S}$ 체계와 연계한 육군 과학화훈련장 확보, 육군 M\&S 국제학술대회 중장기 발전과제가 추가되 어 총 13 개 과제를 선정하였고, 전력지원체계 VV\&A 업무 정립 등 세부 추진사항에 반영하였다. 또한, 본 연구는 국방환경 변화와 해외 M\&S 발전 및 활용 등(Figure 2)을 통한 상황평가를 실시하 고, 이를 토대로 육군의 비전과 목표, 발전과제를 선정하여 M\&S 기본방향을 설정하고, 훈련 M\&S 체계 확보 등 육군 $\mathrm{M \& S}$ 발전방향을 제시하고자 한다.

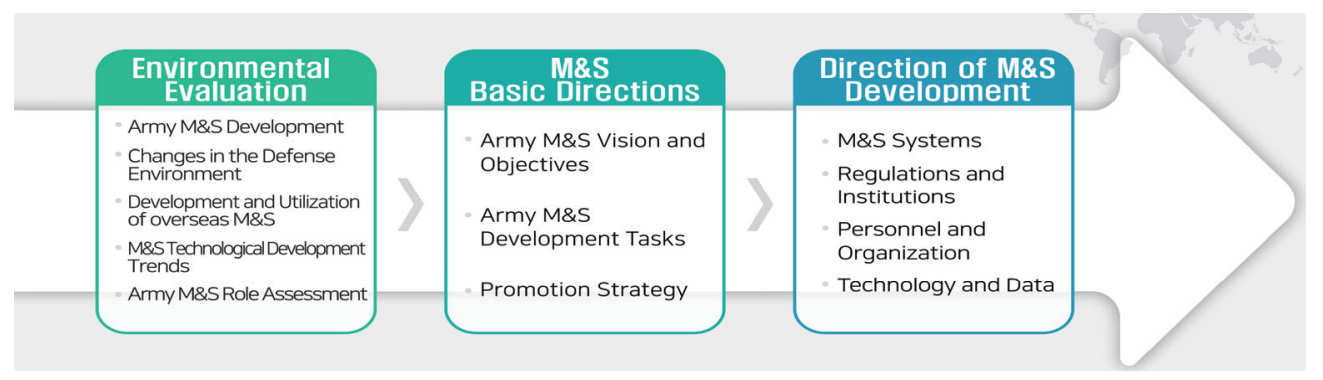

$<$ Figure 2> Army M\&S Development Direction Constitution

\section{II. 상황평가}

\section{1 국방환경 변화에 따른 $\mathrm{M \& S}$ 역할의 증대}

국내외 안보환경은 초불확실성의 시대에 직면하고 있다. 전략환경의 불확실성이 점차 커지고 있 으며, 주변국과의 전력 차이도 점차 심화되고 있다(Kim \& Lee, 2008). 그래서 국방개혁 2.0 및 4차 산업혁명에 따른 시대적 환경변화에 적절한 대응이 필요하며, 예산 삭감의 압력 증대가 예상됨에 따라 육군의 경제적 - 효율적 군사력 건설 및 운영을 위한 방안 수립이 요구된다. 또한, 다양한 과 학 - 기술 분야에서 변화가 빠른 속도로 진행되고 있어 이에 대응하기 위한 정책을 추진하여야 한 다. 이런 환경에 직면한 육군의 변화에 대한 정치, 경제, 사회 - 문화, 기술 분야별로 거시적 환경분 석(PEST, Political Economic Social-Culture Technological)을 살펴보면 다음과 같다(Figure 3).

육군은 분야별 환경변화에 적절한 대응을 위해 병력 의존적 조직에서 첨단과학기술군으로 변모 하기 위해 4 차 산업혁명의 주요 기술을 접목하고, 민 - 관 · 군 - 산 - 학 - 연과의 협업 환경 구축을 통해 창출된 혁신적 기술과 개념을 미래 지향적 군사력 건설에 노력하고 있다. 첨단과학기술군은 미래형 첨단 플랫폼을 갖추고 실시간 초연결 - 지능화된 조직으로 변모하여 다영역 전장을 지배하 는 디지털 육군을 의미한다. 첨단과학기술군 육성을 위해서는 미래 무기체계, 군구조, 작전계획 발 
Future and development ... / Kyoungchan Won • Yong-Pil Kim • Minsuk Kim • Taegyun Ha • Yong-Yeon Lee • Hojun Lee 41

전, 주요정책 수립을 과학적으로 검증하고 소요를 예측해야 하므로 $\mathrm{M \& S}$ 의 활용이 증대될 것으로 판단된다.

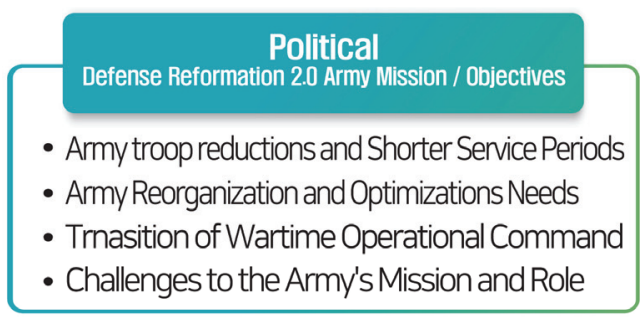

Social-Culture

Changes in Defense Conditions, Enemy Threats, and War Patterns

- Changes in the War Asymmetric Threats fromNK

- Increased Non-Military Threats suchas Terrorismand Cyber

- Massive Reduction of troops

\section{Economic}

Army Budget, Mid to long term Plan

- Restrictions on Defense Budget Growth

- Need to Management Cost Reduction

- Increased Demand for Cost Effectiveness Analysis of NewWeapon Systems

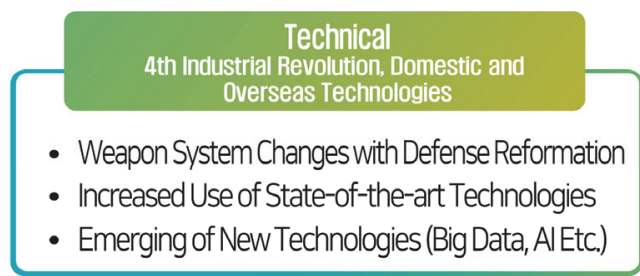

$<$ Figure 3> Summary of PEST analysis of confronting external threats and opportunities changes

이러한 의미에서 $\mathrm{M} \& \mathrm{~S}$ 는 육군이 첨단과학기술군으로 도약하기 위한 핵심수단인 것이다. 여단급 훈련의 패러다임을 바꾼 과학화전투훈련(KCTC, Korea Combat Training Center), 모의논리 기반 의 전투지휘훈련(BCTP), 각종 시뮬레이터를 활용한 전술 및 조종 훈련, 한미연합연습 및 태극연습 등이 M\&S를 통해 이뤼지고 있다(Bang \& Lee, 2020). 훈련 분야 이외에도 작전계획 분석, 전시 자 원소요분석, 무기체계 소요 타당성 분석 등을 통해 각종 계획의 신뢰성을 높이고, 군 운영의 효율성 제고와 새로운 개념 및 무기체계의 타당성을 검증하는데도 M\&S를 활용하고 있다. 이렇듯 M\&S는 육군의 자원을 효율적으로 활용하고, 현실적인 어려움을 극복할 수 있게 해 줄 것이며, 방위력 개선 과 전력증강, 전투준비태세 강화와 미래 과학화훈련 등 효율적인 국방경영의 필수 요소로 활용될 것이다. 미래 다양한 업무수행을 위해 군·산 - 학 - 연 협력을 통한 국방 ICT(Information and Communication Technologies) 신기술 R\&D 사업을 ' 12 년부터 추진하여 점차 확대하고 있으며, 미 래 M\&S 전력소요 및 기술업무 발전으로 군 - 산 · 학 - 연 협력 요구가 증대되고 있다.

\section{2 해외 $\mathrm{M} \& S$ 발전 및 활용}

4차 산업혁명에 따른 ICT의 비약적 발전으로 민간기술이 군의 기술을 크게 앞서 나가고 있는 추세이다. 미국, 영국 등의 선진국을 중심으로 최신 ICT 기술을 군사 목적으로 활용하기 위한 많은 연구 및 개발이 진행되고 있고, 시대적 변화에 맞는 군사혁신을 위해 최신기술에 대한 이해와 이를 $\mathrm{M} \& \mathrm{~S}$ 분야에 적용하는 노력이 중요하게 요구되고 있는 시점이다. 
미군은 훈련, 실험, 획득 분야에 광범위하게 사용 가능한 OneSAF(One Semi-Automated Forces) 개발을 통해 합동작전 모의지원기술을 확보하였고 MetaVR社는 지리에 따른 위치별 지형정보를 $3 \mathrm{D}$ 시뮬레이션 및 가상현실 시뮬레이션이 가능한 기술을 확보하였다. 실제 전장 환경 기반의 증강 현실 모의훈련 장비를 육 - 해 - 공군의 가상훈련에 활용하고 있으며, 육군은 가상증강현실 및 빅데 이터를 활용한 훈련체계 구축으로 합성전장 훈련환경에서 다영역 전투훈련을 추진하고 있다. 미래 사령부는 상용기술을 활용하여 분대에서 군단급 이상 전 제대의 시뮬레이션 훈련이 가능한 합성훈 련환경(STE, Synthetic Training Environment) 개발을 추진하고 있다(Lee, 2020). 미 공군은 전투 훈련체계 중 T-1A 가상훈련 시 MetaVR社의 가상영상 툴(VRSG, Virtual Reality Scene Generator)을 적용한 혼합현실(MR, Mixed Reality) 내장형 시스템을 사용하고 있다.

영국군은 유럽 시뮬레이션 기관인 NMSG(NATO Modeling and Simulation Group)에 참여하고 있으며, 독자 연동 프레임워크 개발, $\mathrm{M} \& \mathrm{~S} \mathrm{DB}$ 구축 및 합성환경 표준화 기술 등을 개발하였고 세 계에서 규모가 가장 큰 가상훈련체계인 제병협동 전술훈련기(CATT, Combined Arms Tactical Trainer)를 운영하고 있다. 또한, LVC 통합훈련의 필요성을 인식하여 예산과 네트워크 등을 고려 하여 장기적 관점에서 연구를 진행하고 있다.

독일은 NMSG에 참여하여 MSaaS(M\&S as a Service), MTDS(Mission Training via Distributed Simulation) 연구 등 M\&S 관련 연구를 수행 중이며, 프랑스는 잠수함, 고정익기 및 회전익기 훈련 시뮬레이터를 활용 중이고, 소부대 전투모델 개발 및 NMSG 활동, 선진국(미국, 영국, 독일)과 기술 교류를 통한 M\&S 기술 확보를 추진하고 있다. 일본은 104J, Kawasaki T4, Mitsubishi UH 60J, Mitsubishi AH 1S 등 항공기 훈련용 시뮬레이터를 개발하여 사용하고 있다. 중국은 가상전투훈련 분야에 대한 투자를 진행하고 있으며 대규모 워게임 시설을 완공하여 교리 - 작전 연구를 추진하고 있다.

\section{III. 육군 $\mathrm{M} \& \mathrm{~S}$ 기본방향}

\section{1 육군 $\mathrm{M} \& S$ 비전 및 목표}

육군 M\&S 비전은 r국방 M\&S종합발전방안 연구 」의 국방 M\&S 비전과 연계하고 상위 정책문 서인 「육군 기본정책서(육군비전2030), 달성에 기여하도록 기능과 역할을 고려하여 Figure 4와 같 이 설정하였다. 한계를 넘어서는 초일류 육군 건설 보장을 위해 $\mathrm{M} \& \mathrm{~S}$ 를 핵심수단으로 활용하여야 한다. 특히 첨단과학기술군으로의 도약을 위해 M\&S의 임무와 역할이 확대되어야 한다. 신뢰성과 효율성이 보장된 M\&S 구현을 위해서는 M\&S 구현에 필요한 제 분야 및 단계의 신뢰성과 효율성 이 보장되어야 한다. 
Future and development ... / Kyoungchan Won • Yong-Pil Km • Minsuk Km • Taegyun Ha • Yong-Yeon Lee • Hojun Lee 43

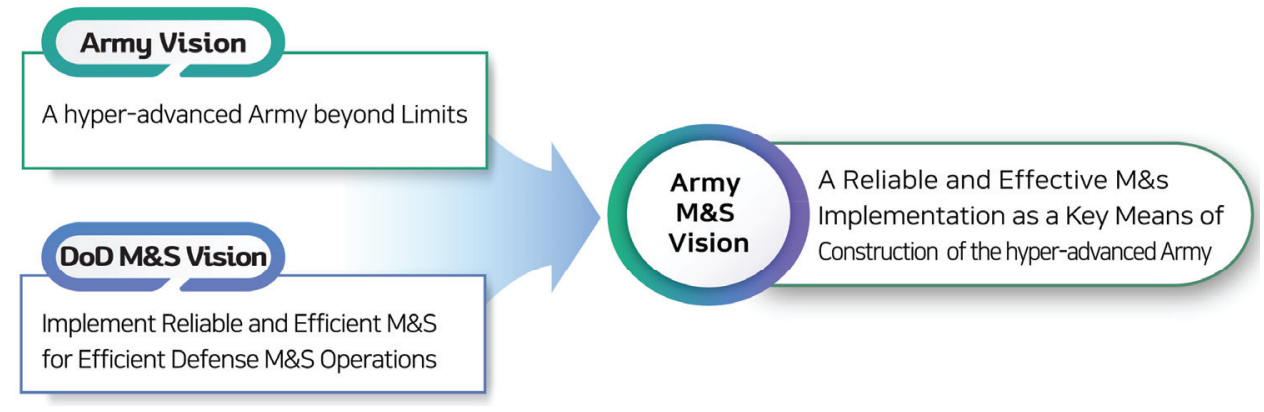

$<$ Figure 4> M\&S vision of Republic of Korea Army

따라서 비전 달성을 위해 '초일류 육군건설의 핵심수단으로서 신뢰성 높고 효율적인 M\&S 구현' 을 위한 요구능력과 이에 따른 분야별 목표를 도출할 필요가 있다(Table 1). 이런 육군 M\&S 목표 는 초안 작성 후에 육군기본정책서를 담당하는 정책실, M\&S 관련 부서인 정보화참모부, 정보작전 참모부 등의 의견을 수렴하여 확정하였다.

$<$ Table 1> Demand capabilities-objectives relationship

\begin{tabular}{l}
\hline \multicolumn{1}{c}{ Demand capabilities } \\
\hline \hline Having an M\&S system, a key scientific means to \\
meet the diverse needs of the Army \\
\hline $\begin{array}{l}\text { Establishment of efficient regulations and } \\
\text { systems underlying M\&S development }\end{array}$ \\
Management of M\&S professionals and \\
organizations
\end{tabular}

Advanced, reliable M\&S technology and data for the Army

\begin{tabular}{l} 
Objectives \\
\hline $\begin{array}{l}\text { Establishment and operation of } \\
\text { M\&S system }\end{array}$ \\
$\begin{array}{l}\text { Improvements to regulations and } \\
\text { systems }\end{array}$ \\
$\begin{array}{l}\text { Training professionals and } \\
\text { organizational development }\end{array}$ \\
Technology and data \\
advancement $\cdot$ standardization \\
\hline
\end{tabular}

\section{2 육군 $\mathrm{M} \& S$ 발전과제 및 추진전략}

육군 M\&S의 비전과 목표달성을 위해 상황평가에 따른 M\&S 역할을 기초로 수행해야 할 발전 과제를 Table 2와 같이 도출하였다. 육군 분석평가단 내 TF를 통해 국방부 M\&S 종합발전방안과 과거 육군 M\&S 발전방향에 대한 연구자료, 육군 기본정책서를 바탕으로 11 개의 육군 M\&S 발전 과제를 선정하였다. $\mathrm{ADD}, \mathrm{KIDA}$, 국방부 등 관련 전문가와 기관의 의견을 수렴하여 Table 2 의 4 번, 11 번 과제인 미래 M\&S 체계와 연계한 육군 과학화훈련장 확보와 육군 M\&S 국제학술대회 중장기 발전과제를 추가하였다. 특히, 육군 M\&S 국제학술대회 중장기 발전은 M\&S 발전을 위해 군 이외 에 M\&S 생태계를 산학연 및 외국까지 확대하여 발전시키는 것이 필요하여 추가하였다. 
$<$ Table 2> Each action plan to achieve the M\&S objectives

\section{Objectives}

I. Establishment and operation of M\&S system

П. Improvements to regulations and systems

III. Training professionals and organizational development

IV. Technology and data advancement • standardization

\begin{tabular}{l}
\hline \multicolumn{1}{c}{ Action plans (Tasks) } \\
\hline \hline (1) Securing training M\&S system to ensure practical training \\
\hline (2) Securing a system for analysis and verification by discharge \\
\hline (3) Development of interlocking system by discharge and \\
function \\
\hline (4) Securing army scientific training center in connection with \\
future M\&S system \\
(5) Improvement of the required raising system according to \\
the characteristics of the M\&S system \\
(6) Development of efficient business cooperation system and \\
expansion of exchanges \\
(7) Acquisition and management of M\&S professionals \\
(8) Improve M\&S training \\
(9) Army M\&S center development \\
(1) M\&S reinforcement \\
(11) Army M\&S international congress mid-to long-term \\
development \\
(1) Establishing M\&S interoperability and standardization \\
(13) Technology and data advancement
\end{tabular}

따라서 육군의 M\&S 정책은 국방부와 합참의 M\&S 정책과 연계성을 유지하여 추진하여야 한다. 국방 $\mathrm{M \& S}$ 종합발전방안연구의 국방 $\mathrm{M \& S}$ 비전과 목표를 공유하며, 중 - 장기 개발이 필요한 육군 $\mathrm{M} \& S$ 체계를 국방 M\&S 종합발전방안연구에 반영한다. 이를 통해 국방부 및 합참과 육군이 동일 한 방향과 내용으로 M\&S 정책을 추진하도록 여건을 보장한다.

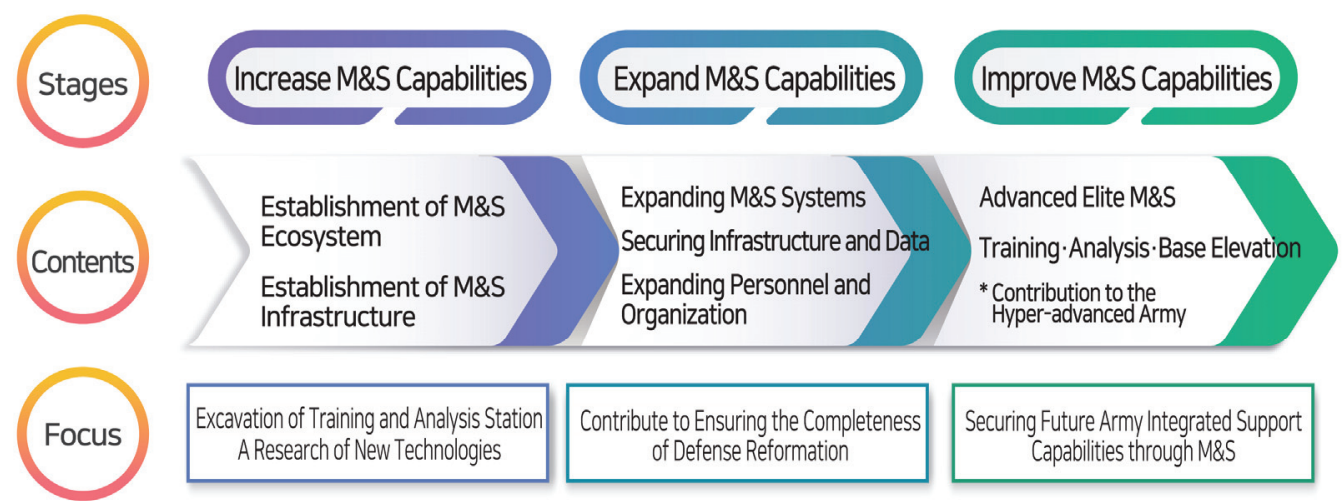

<Figure 5> Key Implementations and goals for each stages in advanced M\&S framework 
Future and development ... / Kyoungchan Won • Yong-Pil Km • Minsuk Kim • Taegyun Ha • Yong-Yeon Lee • Hojun Lee 45

육군의 M\&S 정책발전을 위해 M\&S 생태계 구축과 기반환경을 구축하여 육군의 M\&S 능력을 향상하고자 한다. 그리고 분석 및 훈련 M\&S 체계를 확대하고 기반체계를 확보하여 M\&S 능력을 확장하며, 향후 M\&S 능력을 고도화하는 3단계로 설정하여 상위부서와 일관성 있고, 통합성을 유 지하여 M\&S 발전을 추진하다(Figure 5).

\section{IV. 육군 M\&S 발전방향}

\subsection{M\&S 체계 구축 및 운용}

전 영역 통합작전, 인간 - 드론봇 - AI가 결합된 유-무인 복합전, 새로운 형태의 비대칭전, 초연 결망을 기반으로 한 초연결 전쟁과 같은 미래 전쟁 양상을 구현하기 위한 M\&S 체계가 필요하다. 이를 위한 실천과제는 다음과 같다.

첫째, 최신 ICT 기술과 미래 전투개념을 반영한 훈련 M\&S 체계를 확보해야 한다. 실기동모의 훈련체계 발전을 위해 현 여단급 과학화 전투훈련체계(KCTC)의 제한사항을 극복하고, 실전성, 확 장성 및 편의성을 향상시키는 미래 과학화 전투훈련체계를 장기 전력소요에 반영해야 한다. 특히 핵심기술 연구를 통해 비레이저 기반의 마일즈 기술을 획득하고 최신기술과 수명 연한을 고려하여 Table 3과 같이 미래 과학화 전투훈련체계를 발전시킨다.

$<$ Table 3> Developmental directions of scientific military training system in R.O.K. Army in the future

\begin{tabular}{|c|c|}
\hline Limitations & Developmental directions \\
\hline $\begin{array}{l}\text { - Damage done in the order of the laser beam } \\
\text { - Restrict damage in laser shielding } \\
\text { - Restrictions for fierce battlefield implementation } \\
\text { - } 1.5 \text { generation-based communication restricts } \\
\text { implementation of battlefield implementation } \\
\text { - Limited federations and joint operations possible }\end{array}$ & $\begin{array}{l}\text { - Non-Laser-based Miles development } \\
\text { - Reflects the latest weapon systems such as integrated } \\
\text { bulletproof helmets and warrior platforms } \\
\text { - Establishment of } 5 \mathrm{G}-\text {-based wireless mobile } \\
\text { environment with high-speed optical communication } \\
\text { network+commercial network } \\
\text { - Building a big data platform }\end{array}$ \\
\hline
\end{tabular}

둘째, 제대별 분석 및 검증체계를 위한 체계를 확보해야 하며, 이를 충족할 수 있는 분석 M\&S 체계는 Table 4와 같이 발전시켜야 한다. 제대별 효율적 부대 운용, 작전계획 수립 및 검증을 위한 분석용 모델을 발전시키고 이를 운용하기 위한 능력을 보강해야 한다. 다양한 무기체계 확보에 따 른 효과를 검증 및 분석할 수 있는 효과분석 모델의 개발이 필요하다. 
$<$ Table 4> Echelon Analysis Requirement (Proposal)

\begin{tabular}{|c|c|c|}
\hline Echelon & Analysis Focus & Analysis Requirement \\
\hline Army HQ & $\begin{array}{l}\text { At all times, analysis to support } \\
\text { the Decision-Making of the } \\
\text { Command team }\end{array}$ & $\begin{array}{l}\text { - Forces \& Unit Structure Verification } \\
\text { - Individual Weapon System Requirement Verification } \\
\text { - Major Business Force Operation \& Cost Analysis } \\
\text { - Main Issue Analysis } \\
\text { - Analysis of Wartime Resource Acquirement } \\
\end{array}$ \\
\hline $\begin{array}{l}\text { Operation } \\
\text { Command }\end{array}$ & $\begin{array}{l}\text { Support to Ground Operation } \\
\text { (Support to Rear Operation) }\end{array}$ & $\begin{array}{l}\text { - Operation Plan Establishment Development Verification } \\
\text { - NK Ground Forces Analysis } \\
\text { - Current Operation } \\
\text { - Future Operation } \\
\text { - CMO (Civil-Military Operations) } \\
\text { - Counter Fire Operation } \\
\text { - MOUT (Military Operations in Urban Terrain) }\end{array}$ \\
\hline TRADOC & $\begin{array}{l}\text { Future DOTMLPF Analysis } \\
\text { /Verification \& Experiment }\end{array}$ & $\begin{array}{l}\text { - DOTMLPF Optimization } \\
\text { - Warfight Experiment } \\
\text { - DOTMLPF Functions Verification }\end{array}$ \\
\hline $\begin{array}{l}\text { Division/ } \\
\text { Corps }\end{array}$ & $\begin{array}{l}\text { Analysis to ensure Corps- } \\
\text { Centered Operational conditions }\end{array}$ & $\begin{array}{l}\text { - Operation Plan Development \& Verification } \\
\text { - Real Time Plan Analysis } \\
\text { - Operation Sustainment Plan \& Verification } \\
\text { - } \text { Current Operation Forecast } \\
\text { - Future Operation Decision } \\
\text { - Unit • Functional Mission Performance Analysis in } \\
\text { Wartime \& Normal }\end{array}$ \\
\hline
\end{tabular}

셋째, 제대별 - 기능별 연동체계를 개발해야 한다. 교육훈련 효과를 극대화하기 위한 다양한 훈 련체계를 동시 - 통합 운용할 수 있는 플랫폼의 발전을 의미한다. 육군은 현재 육군합성전장훈련체 계 개발사업을 통해 LVC 연동훈련체계를 2026년에 전력화할 예정이다. 개발된 LVC 연동훈련체계 를 통해 $\mathrm{KCTC}$, 육군항공 시뮬레이터, $\mathrm{UAV}$ 시뮬레이터, 사 - 군단급 워게임모델인 창조21모델을 연 동하여 사단급 LVC 통합훈련을 발전시킨다. 여단급 이하는 STE(Synthetic Training Environment) 플랫폼으로 통합하고 초연결 - 초지능을 달성하여 24 시간 언제, 어디서나, 다양한 훈련이 가능하도 록 훈련체계 구축을 추진한다.

넷째, 미래 M\&S 체계와 연계한 육군 과학화훈련장을 확보해야 한다. 미래 육군의 과학화훈련 발전과 연계한 LVCG 훈련장을 구축하고, 실기동훈련장은 소대급 이하 소부대 훈련장과 중대급 이 상 권역화 훈련장으로 구분하여 구축한다. 제병협동 권역화 전술훈련장은 중대급 이상 제대가 제병 협동 실기동 전술훈련, 편제화기 사격 및 전투사격, 가상모의훈련을 동시에 할 수 있도록 훈련장을 구축하여 일정한 지역 내 부대가 공동으로 사용한다. 2작전사 권역 훈련장은 지역방위사단 대대급 이상 부대가 상시 훈련이 가능한 종합훈련장을 구축한다. 
Future and development ... / Kyoungchan Won • Yong-Pil Kim • Minsuk Kim • Taegyun Ha • Yong-Yeon Lee • Hojun Lee 47

\section{2 규정 및 제도 개선}

변화하는 국방환경에서 효율적인 M\&S 체계 획득을 위해 관련 규정과 제도의 발전이 필요하다. 다양한 M\&S 체계 소요제기 제도 활용하기 위해 M\&S 소요창출 프로세스를 개발하였다(Figure 6). 소요창출 기본 개념은 소요기획문서체계와 연계하여 소 단계 프로세스를 설계하는 것으로서 '(1) 소 출처 소요 염출 $\rightarrow$ (2) 소요 목록화 $\rightarrow$ (3) 소요창출 $\rightarrow$ (4) 소요반영 $\rightarrow$ (5) 예산반영 $\rightarrow$ (6) 사업 관리'의 절차를 구체화하는 것이다. 이를 구체적으로 설명하면, 우선 소 출처(현용-창출) 소요를 염출하여 기관별 소요기획문서와 로드맵을 통해 구체화한다.

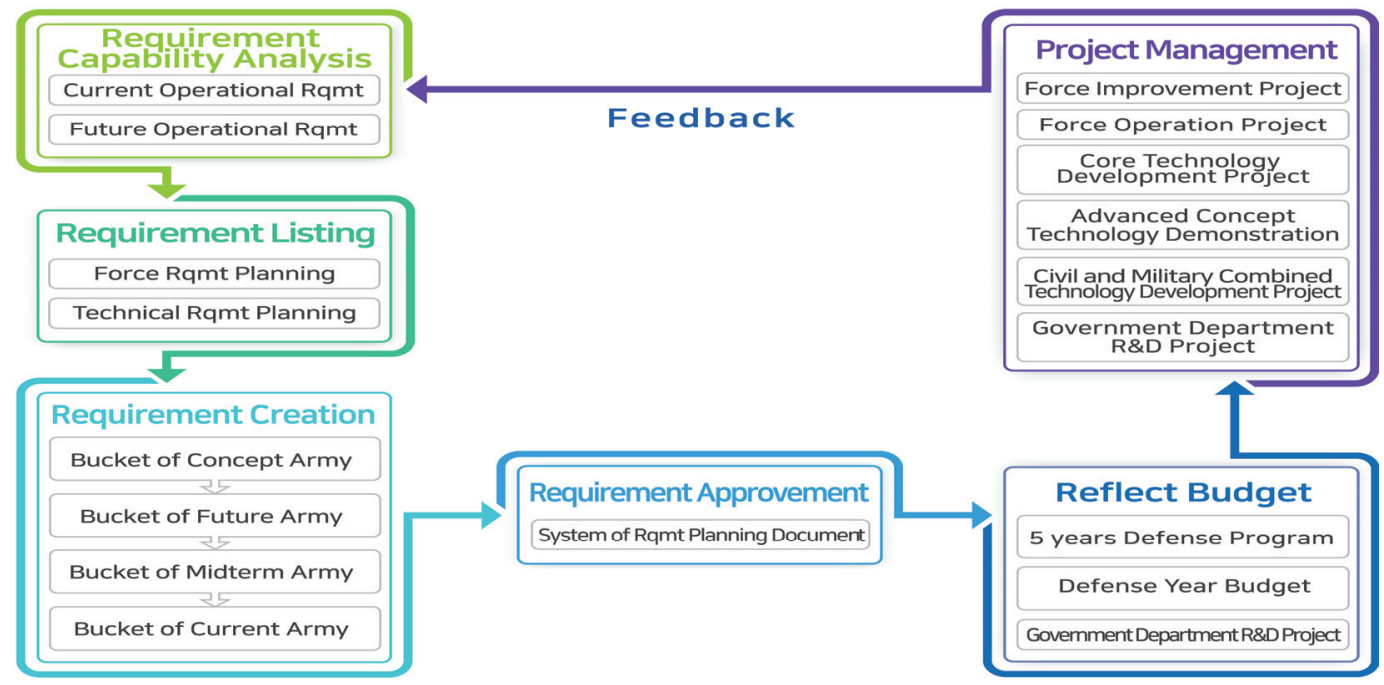

$<$ Figure 6> M\&S Requirement Process

다음으로 M\&S 생태계를 활용하여 미군, 이스라엘 등 선진군이 적용하고 있는 과학기술을 발굴 하여 미래 전력소요와 기술 소요를 목록화한다. 이후, 목록화된 기술을 4 개의 첨단기술 바구니 (Bucket)인 '개념군(Bucket of Concept), 미래군(Bucket of Future), 중간군(Bucket of Midterm), 현 용군(Bucket of Current)'2)에 담고, 시기별 전력화에 필요한 소요를 창출한다. 이어서, 과학기술 분 과별 분류에 대한 적절성 검토와 소요의 우선순위를 조정하여 소요기획문서체계에 소요를 반영하 고, 국방예산과 정부 부처 $\mathrm{R} \& \mathrm{D}$ 사업 예산으로 구분하여 사업을 관리한다.

2) 개념군(30년 정도의 초장기 미래), 미래군(10년 정도의 장기 미래), 중간군(5년 정도의 중기 미래), 현용군(현재 및 가까운 미래) 




$<$ Figure 7> Example of VV\&A

$\mathrm{M \& S}$ 체계의 신뢰성과 재사용성을 높이고 표준화를 수행하기 위해 Figure 7과 같이 M\&S 인정 (VV\&A : Verification Validation and Accreditation) 업무수행 체계의 정립이 필요하다(Yoo, Kim, Lee, \& Kim, 2020). VV\&A는 M\&S 개발과 동시에 시작되며 요구사항 구체화, 개념모델링, 설계, 구현, 시험평가 등 전 단계에서 시행한다. 또한, 모의논리, 데이터, 운용 등 전 분야를 통해 최종 인 정을 수행한다(Han, 2021). 특히, 효율적 업무협력 체계 정립을 위해 기관별 M\&S 업무수행 체계를 정립하고, 육군 $\mathrm{M} \& \mathrm{~S}$ 발전 업무를 위해 국방부, 합참, 연합사, $\mathrm{ADD}, \mathrm{KIDA}$ 등 $\mathrm{M} \& \mathrm{~S}$ 관련 기관과의 유기적인 협조 관계를 구축해야 한다.3) 육군 참모부 및 M\&S 관련 부서의 수행업무(안)은 Table 5 와 같이 제안한다.

국방 M\&S 차원에서 개발 전 단계에 걸친 $\mathrm{VV \& A}$ 규정을 정립하고 수행하는 기관과 역할, 산 출물 등을 구체화해야 한다. 육군은 이를 바탕으로 개발단계 중 사용자로서 육군 M\&S 센터를 중심으로 기획관리 전 단계에서 VV\&A 수행에 필요할 역할을 식별하고 이를 위한 인력/조직을 확보하는 등 VV\&A 업무수행 능력을 구비하고 업무분장을 구체화한다. 장기적으로는 무기체계 $\mathrm{M} \& \mathrm{~S}$ 뿐만 아니라 국방정보화시스템 및 교육훈련물품 등 전력지원체계 M\&S 개발 간에도 $\mathrm{VV \& A}$ 를 적용하여 M\&S 신뢰성 확보를 추진하며, 육군의 전력지원체계 VV\&A 업무분장(안)은 Table 6과 같다. 
Future and development ... / Kyoungchan Won • Yong-Pil Kim • Minsuk Kim • Taegyun Ha • Yong-Yeon Lee • Hojun Lee 49

$<$ Table 5> M\&S Performance by Institution (Proposal)

\begin{tabular}{|c|c|c|}
\hline \multicolumn{2}{|c|}{ Institute } & Performance \\
\hline \multirow{5}{*}{$\begin{array}{c}\text { Army } \\
\mathrm{HQ}\end{array}$} & G5 & $\begin{array}{l}\text { - Weapon System M\&S Requirements } \\
\text { - Weapon System Verification }\end{array}$ \\
\hline & G2 & $\begin{array}{l}\text { - Establishment of Training M\&S Operation Concept } \\
\text { - Training M\&S Requirements (Force Support System Project) }\end{array}$ \\
\hline & G1 & - M\&S Cooperation in the Development of Professional Manpower \\
\hline & G6 & $\begin{array}{l}\text { - Force Support System M\&S Take Raise } \\
\text { - Interoperability Task Cooperation }\end{array}$ \\
\hline & G4 & $\cdot$ M\&S Development ILS \\
\hline \multirow{3}{*}{$\begin{array}{l}\text { Research } \\
\text { Institute }\end{array}$} & KIDA & $\begin{array}{l}\text { - Foreign M\&S Technology, Data Cooperation Support } \\
\text { - Foreign Adopt/Development M\&S Operation Support }\end{array}$ \\
\hline & $\mathrm{ADD}$ & $\begin{array}{l}\cdot \text { SBA Cooperation Center } \cdot \text { ROC Verification } \cdot \text { M\&S Core } \\
\text { Technology Development }\end{array}$ \\
\hline & $\mathrm{DTaQ}$ & $\cdot$ - M\&S Data Standardization $\cdot$ VV\&A $\cdot$ HLA Certification \\
\hline \multirow{4}{*}{$\begin{array}{l}\text { Upper/ } \\
\text { Exterior } \\
\text { Institute }\end{array}$} & $\mathrm{CFC}$ & $\cdot$ Combined Exercise \\
\hline & MND & $\begin{array}{l}\text { - M\&S Policy Regulation Development } \\
\text { - MND M\&S Budget } \\
\text { - Force Support System of M\&S Requirement Decision } \\
\text { - National Defense Reform } \\
\text { - National Defense Mid-Term Plan }\end{array}$ \\
\hline & JCS & $\cdot$ M\&S Budget Cooperation $\cdot$ Joint Analysis/Experiment $\cdot$ Joint Exercise \\
\hline & DAPA & - Weapon System M\&S Project Management \\
\hline \multirow{2}{*}{$\begin{array}{l}\text { Army } \\
\text { Sub. }\end{array}$} & TRADOC & - M\&S Requirement Suggestion \\
\hline & $\begin{array}{l}\text { Operation } \\
\text { Command }\end{array}$ & • M\&S Requirement Suggestion \\
\hline
\end{tabular}

$<$ Table 6> R.O.K. Army Force of Support System VV\&A Tasks (Proposal)

\begin{tabular}{|c|c|}
\hline Institute & Performance \\
\hline Project Parent Station & $\begin{array}{l}\text { VV\&A Task Mediation/Control } \\
\text { - Accreditation Role } \\
\text { - VV\&A-related budget reflected in Mid-term • Annual Budget }\end{array}$ \\
\hline Project Management Station & $\begin{array}{l}\text { - Overall VV\&A Task Management and Direction } \\
\cdot \text { V\&V Agent Approvement, Accreditation Agent Designation } \\
\text { *V\&V Agency Selected from Project Parent Station }\end{array}$ \\
\hline CAAS & $\begin{array}{l}\text { - V\&V Activity Support (SME) } \\
\text { - Army SME Management }\end{array}$ \\
\hline TRADOC & - V\&V Activity Support (SME) \\
\hline
\end{tabular}




\section{3 전문인력 양성 및 조직 발전}

4차 산업혁명과 국방개혁 등의 환경변화 속에서 육군의 도약적 변혁을 선도하기 위한 다양한 분 야의 업무에 대하여 객관적인 분석과 과학화 훈련 등을 지원하기 위한 조직과 전문인력이 확보되 어야 한다. 또한, 전작권 전환대비 육군의 군사력 건설, 군구조 개편, 교육훈련 등을 주도할 M\&S 전문인력의 육성이 필요하다(Han, $\mathrm{Kim}, \& \mathrm{Jae}, 2012)$. 전문인력 양성 및 조직발전을 위해 추진할 세부과제는 다음과 같다.

첫째, 우수한 M\&S 인력을 획득하고 관리하여야 한다. 육군은 우수자원을 선발하여 국방관리분 석 특기를 부여하고, 전문학위 및 군사교육, 직무향상교육, 전문직위 보직, 야전순환 등 다양한 보 직을 경험하여 전문지식과 야전감각을 동시에 갖춘 $\mathrm{M \& S}$ 전문인력을 양성한다. 실무와 연계된 교 육과 다양한 직위를 경험하여 M\&S 전문가로 육성하고 활용해야 한다. 또한, M\&S 업무 경험이 풍 부한 예비역의 활용을 확대하고, 전문지식과 경험을 보유한 전문분석관을 확충하여 부족한 인력을 보완해야 한다. 둘째, M\&S 교육개선을 위해 야전과의 공감대를 형성하고, 의사소통 활성화를 추진 한다. 이를 위해 장교양성 및 보수교육 과정에 M\&S 이해 및 활용 교육이 Table 7과 같이 확대 반 영되어야 한다. 양성교육과 보수교육 과정에 제대별 수준별 맞춤형 M\&S 교육을 실시하여 M\&S 기본 개념과 활용의 필요성을 이해하고 향후 발전에 대한 공감대를 형성한다.

$<$ Table 7> Education plan for defense M\&S

\begin{tabular}{|c|c|c|c|}
\hline \multicolumn{2}{|c|}{ Criteria } & Role & Note \\
\hline \multirow{3}{*}{$\begin{array}{l}\text { Training \& } \\
\text { Education }\end{array}$} & KMA & $\begin{array}{l}\text { - Simulation(Major 1st Class, 3H/1Week) } \\
\text { - M\&S(Common 1st Class, 3H/1Week) }\end{array}$ & \multirow{3}{*}{$\begin{array}{l}\text { Included in the } \\
\text { common curriculum }\end{array}$} \\
\hline & KTMA & · M\&S(Common 1st Class, 3H/1Week) & \\
\hline & ROTC & · M\&S Introduction(Common, 2H/1Week) & \\
\hline \multirow{5}{*}{$\begin{array}{c}\text { Conservative } \\
\text { Education }\end{array}$} & $\mathrm{OAC}$ & - M\&S Introduction & \multirow{5}{*}{$\begin{array}{l}\text { Conducted level } \\
\text { differential education in } \\
\text { common curriculum }\end{array}$} \\
\hline & Major Basic & $\cdot$ CBSC \& JWSC Tour & \\
\hline & Major Special & - M\&S Understand \& Use & \\
\hline & LTC & - M\&S Institution & \\
\hline & Colonel & - M\&S Policy & \\
\hline
\end{tabular}

셋째, M\&S 정책발전, 소요기획 업무를 추진하기 위해 육군 M\&S 센터를 발전시켜야 한다. 육군 분석평가단 예하 M\&S 센터장 직위 신설을 중기로 추진한다. 장기적으로 분석평가단을 3 개처로 개 편하여 육군 M\&S 센터 혹은 M\&S처 형태의 별도 조직을 편성하고 계획된 편제인원을 확보 후 육 군 M\&S 센터의 고유 기능을 수행한다. 또한, 계룡대 통합분청 신축계획과 연계하여 육군전쟁지원 통합시설(AWSC, Army Warfighting Simulation Center)을 추진한다(Figure 8). 
Future and development ... / Kyoungchan Won • Yong-Pil Kim • Minsuk Kim • Taegyun Ha • Yong-Yeon Lee • Hojun Lee 51

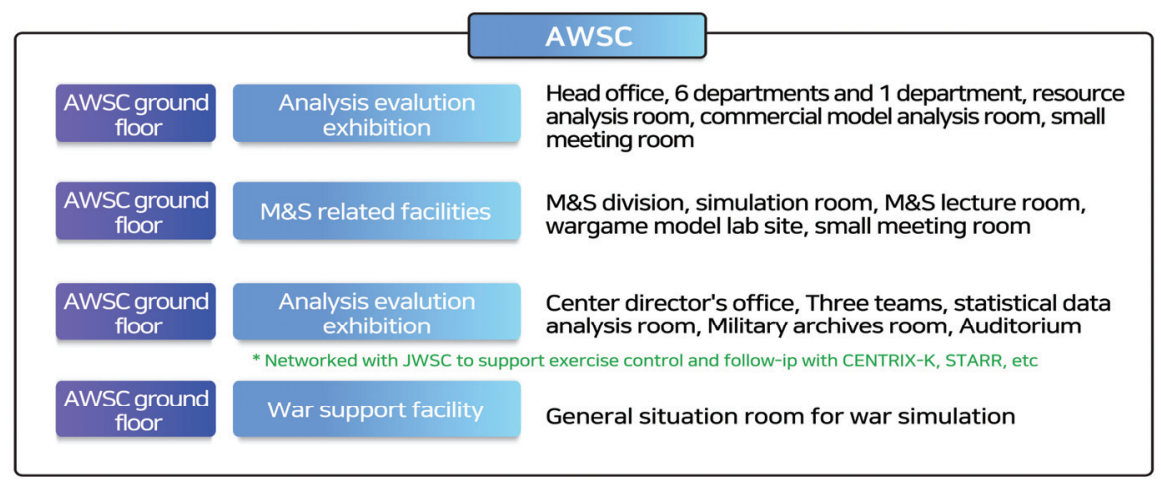

<Figure 8> AWSC Configuration (Proposal)

넷째, 국방 M\&S 발전을 위해 조직 보강이 필요하다. 분석 M\&S 조직 보강을 위해 지상작전사령 부 작전분석과의 임무와 역할을 고려하여 제 2 작전사령부 예하에 과 규모의 분석조직을 신편하여 작전지속지원계획 및 통합방위작전 수립을 검증 및 지원하고 운영 효율성 보장을 위한 분석업무를 지원한다. 훈련 M\&S 조직 보강을 위해 지상작전사령부 예하 군단별 다양한 분석소요를 충족하기 위한 군단 작전처 예하에 분석인원을 증편하여 작전계획 수립, 방책분석 등의 실전적 전투 임무수 행을 지원한다. 교육사의 교육훈련부를 통해 향후 확대 발전되는 사· 군단급 과학화 훈련체계 구 축에 대한 통제와 효과분석 및 기술연구 등의 관리, 소요기획부터 획득 등의 임무를 수행하기 위해 과학화훈련센터 편성을 추진하고, 육군 과학기술위원회 LVCG(Live, Virtual, Constructive, Game) 기술그룹 운영을 담당하여 육군 과학화 훈련체계의 통제 및 운영을 통해 LVCG 기반 과학화 훈련 체계 발전을 주도한다. 육군 M\&S 기술연구 조직은 육군 M\&S 센터 발전과 병행하여 M\&S 발전과 를 보강하여 그 기능을 수행하고 동시에 육군의 기술기획 업무를 담당하는 육군미래혁신센터 기술 융합과와 협업을 통해 그 기능을 확대 발전시킨다. 기술관리 조직 보강은 육군 $\mathrm{SW}$ 관리업무체계 발전을 고려하여 단기적으로 SW 관리업무와 통합하고, 장기적으로는 육군에서 활용되는 시뮬레이 터를 포함한 모든 M\&S 체계의 데이터 개발, 관리, 보완 등을 수행하는 조직을 신설하여 관리한다.

마지막으로 육군 M\&S 국제학술대회는 국제학술대회로서의 위상을 갖추기 위해 해외 여러 국가의 관련 기관이 함께 참여하여 각 나라의 발전동향을 공유하고 최신기술 활용을 위한 공감대를 형성해 왔다. 지난 학술대회 동안 합동군사대학 및 국방대학교의 해외 수탁장교와 주한 무관부의 국방무관 그 리고 미국, 영국, 독일, 아시아 등의 관련 기관이 참가하였다. 특히 미 육군 분석평가국(CAA, Center for Army Analysis)와 영국 지상군은 정기적인 참석으로 국제행사로서의 위상을 제고할 수 있었다. 학술대회의 규모를 확대하기 위해 공동주최기관인 육군, 대전광역시, 국방과학연구소를 넘어서 누구 나 참여하고 공감할 수 있도록 온택트 형식의 학술대회를 병행하여 추진한다. 이는 실시간 스트리 밍 방송형식을 통해 유튜브를 통한 행사 생중계, 페이스북 등의 SNS 매체를 통하여 언제 어디서나 현장의 생동감을 느끼며 참여할 수 있도록 발전해야 한다. 이러한 발전을 확대하기 위해'23년에 실 
시하는 제 16 회 학술대회는 유럽 NATO의 M\&S 관련 기관인 NMSG(NATO Modeling Simulation Group)를 국내로 초대하여 NMSG의 정기회의(BM, Business Meeting)를 공동으로 주최하는 것으 로 추진하고, 이러한 성과를 바탕으로 '23년 이후 NMSG와 정기적인 교류행사를 추진하여 미국 외 에 다양한 유럽국가와 M\&S 관련 기술을 공유하고 발전방향에 대한 공감대를 형성하여야 한다.

\section{4 기술과 데이터의 고도화 및 표준화}

분산환경에서 M\&S 체계의 효율적 운용을 위한 상호운용성이 확보되어야 한다. M\&S 획득을 위 한 상호운용성 업무체계를 정립하기 위해 소요기획 단계에서는 연동소요를 식별하고, 목표로 하는 연동수준을 결정하며 연동대상체계 간 연동합의서를 체결하고, 체계개발 단계에서는 요구사항(연 동소요)을 구체화하고 요구사항대로 구현하도록 지속적으로 확인한다. 시험평가 단계에서는 상호 운용성 평가를 지원하고 연동표준(HLA4) 인증을 획득한다(Hong, Sung, Ahn, \& Kim, 2009). $\mathrm{M} \& S$ 체계개발 시 국방 M\&S 상호운용성 표준 적용을 추진하기 위해 국방 아키텍처 프레임워크 표준절차를 활용하고, 개발 시 국방 $\mathrm{CBD}^{5}$ 방법론을 적용하여야 하며, 표준 부대코드와 부대부호 를 적용한다. 또한, 타 체계와의 연동을 위한 국방 표준연동규약을 준수한다.

$\mathrm{M} \& \mathrm{~S}$ 를 통한 모의결과의 신뢰성을 향상시키기 위해 M\&S 체계 입력 데이터의 표준화 및 고도

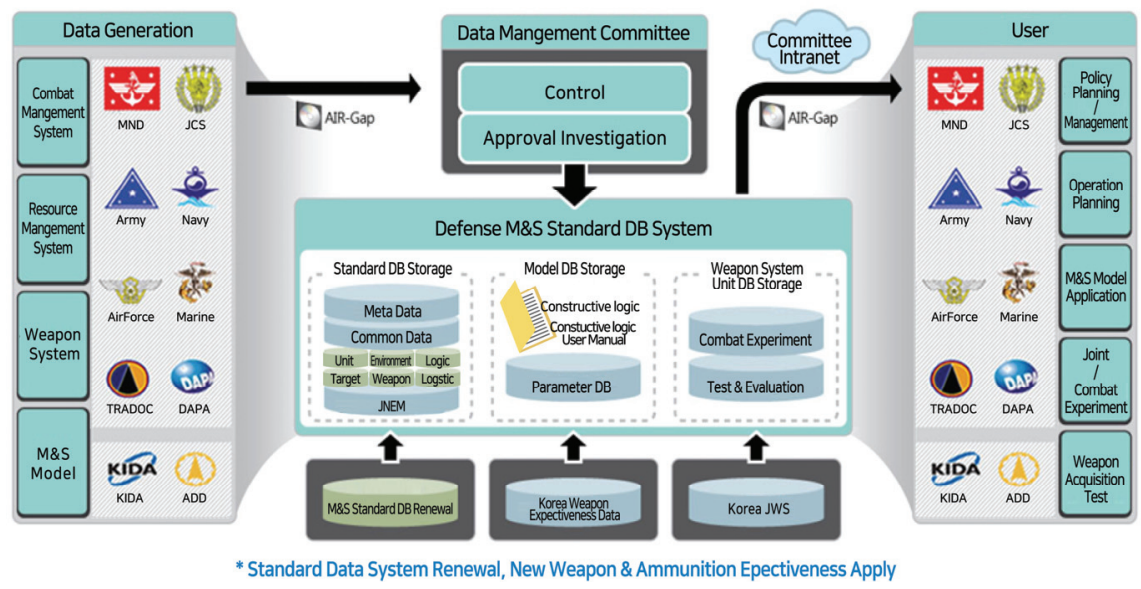

$<$ Figure 9> Framework of the proposed standard data system

* Standard Data System Renewal by apply New Weapon and Ammunition Effectiveness

4) High Level Architecture : 연동표준기술구조. 분산 처리 컴퓨터 시뮬레이션 시스템을 위한 범용 아키텍처로 컴퓨터 시뮬레이션이 컴퓨터 플랫폼에 상관없이 다른 컴퓨터 시뮬레이션과 연결이 가능

5) Component Based Development : 시스템을 하나의 일체형으로 구축하지 않고 요소별 부품화하여 구축하는 방법 
Future and development ... / Kyoungchan Won • Yong-Pil Kim • Minsuk Kim • Taegyun Ha • Yong-Yeon Lee • Hojun Lee 53

화를 추진해야 한다. 합참 차원의 JWS6) 도입사업을 통해 획득한 신뢰성 있는 데이터를 활용하여 무기효과 데이터를 표준화하고 M\&S 체계에 적용함으로써 결과물에 대한 신뢰도를 향상시킨다. 품질 높은 데이터를 생성하여 M\&S를 통한 모의결과의 신뢰성 향상에 기여하기 위해 Figure 9와 같이 표준자료체계를 구축을 추진해야 한다.

전장관리체계의 데이터 및 전장상황을 분석하여 지휘결심을 지원하는 인공지능 기술 기반의 지 휘결심지원체계를 발전시킨다. 인공지능 기술 기반 지휘결심지원체계는 다음과 같이 2 단계로 인공 지능을 고도화시킨다. 1단계는 빅데이터 학습을 통한 상황별 방책 수립 방법 모델링으로 JOAM-K (Joint Operations Analysis Model-Korea) 등과 같은 분석용 모델에서 운용자가 매 전술적 상황에 어떻게 대처하였는가에 대한 궤적(trajectory)을 학습하여 다양한 전장 상황에 대한 방책 수립 방법 을 학습하게 되며, 이렇게 학습된 내용을 활용하여 방책 수립 방법을 모델링한다. 2 단계는 강화학 습 알고리즘 기반 진화적 지휘결심지원체계 모델링으로 반복적인 시뮬레이션을 통하여 다양한 전 장 상황에서 행동에 대한 보상을 최대화할 수 있도록 자신의 행동 규칙을 스스로 진화시킬 수 있는 지휘결심지원 체계를 모델링 한다. 이를 통해 기존의 규칙기반 학습과 같이 지속적인 관리를 하지 않더라도 항상 최신성 유지가 가능하다. M\&S 위해 표준화 체계 구축과 한반도 작전환경 및 전쟁 양상 변화에 부합된 모의논리를 고도화해야 한다. M\&S에 대한 공통 모의논리 체계를 구축하기 위 해 모델별로 산재되어 있는 모의논리를 기능 및 내용별로 표준화하여 관리할 수 있도록 기준을 정 립하고 모의논리서 작성 및 국방 M\&S 표준자료체계 관리에도 적용한다. 병과별 군 전문가들이 연 계 모의논리를 추적할 수 있게 함으로써 교리 또는 전투경험을 통해 전쟁 수행 방법 및 무기체계 변경 내용이 지속 보완될 수 있도록 한다.

마지막으로 부대훈련과 운영데이터의 수집 및 관리체계를 Figure 10과 같이 구축하여야 한다. 훈련데이터 및 부대운영 데이터를 체계적으로 수집 - 관리하여 육군의 전투력 발전, 정책 의사결정

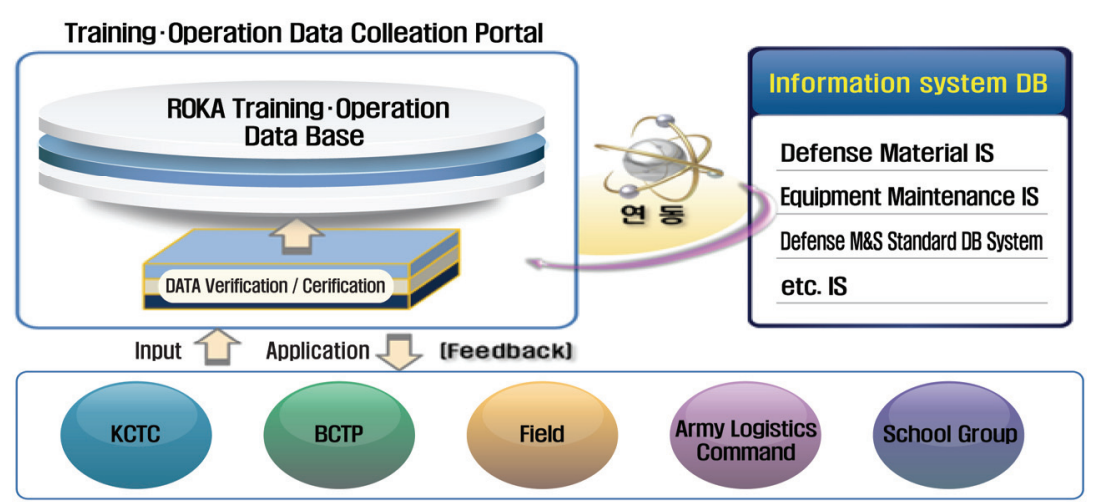

$<$ Figure 10> Concept diagram of data collect and construction from training \& unit operation

6) JMEM Weaponeering System : 군사작전 분석, 전력소요 분석 등을 위해 ⿱䒑夫 합동무기효과기술조정단(JTCGME)에서 지난 40여 년 동안 개발한 공대지 및 지대지 무기체계의 무기효과자료와 무기효과분석도구를 수록한 통합 소프트웨어 패키지 
지원, M\&S 체계 발전을 위한 기초데이터 활용체계를 구축한다. 이를 통해 신뢰성 있는 데이터를 수집, 구축, 분석하여 육군 사업의 기초자료로 활용하고 작전계획 수립 지원 및 육군 운영 효율화에 활용하며, M\&S 훈련 결과로부터 전훈 분석에 활용한다.

Table 8은 육군이 수집해야 하는 데이터 대상이다. 훈련 및 부대운영 데이터를 수집하여 신뢰성 있는 기초자료를 제공함으로써 육군의 교리발전 및 교육훈련 등 전투발전에 지원이 가능하다(Jeon, $\mathrm{Kim}, \&$ Lee, 2020). 특히, 한반도 전장 환경에 부합된 자료 획득이 가능하고, 교리발전 및 교육훈련 발전을 지원하는 다양한 자료를 지원할 수 있다.

$<$ Table 8> M\&S Data Collect Target (Example)

\begin{tabular}{l|l}
\hline \multicolumn{1}{c|}{ Criteria } & \multicolumn{1}{c}{ Contents } \\
\hline \hline Education & Unit $\cdot$ Class $\cdot$ Season Training Data, DOTMLPF \\
\hline LVC Training & KCTC, BCTP, Simulator, Battle Test \\
\hline Unit Operation & Maintenance, Transport, Supply, Standard Hour, etc. \\
\hline Weapon System & Test Evaluation, Basic Specification, Oil Consumption Rate, etc. \\
\hline Battlefield Information/Operation & Terrain, Weather, Counter-Terrorism Operation, Deployment \\
\hline
\end{tabular}

\section{V. 결론}

육군은 최근 훈련장 부족, 재정의 압박, 민원의 증가 등 국방환경이 변화함에 따라 훈련체계 및 의사결정을 지원할 수 있는 새롭고 획기적인 도구가 필요하며, 병역자원의 감소와 복무기간의 단축 추세에 따라 단기간에 훈련 효과 달성이 가능한 훈련수단이 요구되었다. 따라서 이러한 문제를 극 복할 수 있는 대안으로 과학화된 훈련 및 분석의 핵심수단인 M\&S는 선택이 아닌 필수 요소이다. 적 위협, 전쟁 양상, 국방여건의 변화 등 환경의 변화 속에서 육군 비전을 구현하는 핵심수단으로서 $\mathrm{M} \& S$ 역할이 중요해짐에 따라 육군 M\&S 발전을 위한 구체적인 정책문서가 요구되었다. 따라서 육군은 M\&S 체계의 발전을 통해 미래 육군을 설계하고 건설하며, 효율적 운영을 보장하는 합리적 인 대안을 만들어 가는데 기여할 것으로 판단된다. 특히, 우리 육군은 미래 변화하는 국방환경에 유연하고 합리적으로 대응하기 위해 다양한 훈련과 분석 소요가 발생할 것이다. 이를 위해 실기동 모의, 시뮬레이터, 워게임 모델별로 신규 및 성능개량을 통해 모의지원 능력을 발전시켜 나가야 하 며, 국방개혁에 따른 제대별 분석을 지원할 수 있도록 제대별 분석능력 향상 등의 다양한 M\&S 체 계를 발전시켜야 한다. 따라서 육군의 모든 사업은 M\&S를 통해 객관적으로 검증되어야 하며 훈련 은 실전적이고 과학적으로 이루어져야 한다.

본 연구는 첫째, 변화한 국방환경을 고려하여 국방 M\&S 종합발전방안」과 연계하고, 19-33 육군 
Future and development ... / Kyoungchan Won • Yong-Pil Kim • Minsuk Kim • Taegyun Ha • Yong-Yeon Lee • Hojun Lee 55

기본정책서인'육군 비전 2030 ‘의 내용을 토대로 육군의 M\&S 정책방향을 제시하였다. 둘째, 변화 하는 국방환경과 해외 M\&S 발전 및 활용을 분석하여 상황을 평가하였다. 셋째, 육군의 M\&S 기본 방향을 설정하여 ‘초일류 육군 건설 구현의 핵심수단으로서 신뢰성 높고 효율적인 M\&S 구현’이라 는 육군 M\&S 비전을 새롭게 도출하였다. 'M\&S 체계 구축 및 운용', '규정 및 제도 개선', '전문인력 양성 및 조직 발전', '기술 및 자료 고도화 - 표준화'라는 4개의 목표를 제시하였다. 변화된 각종 규 정 및 제도와 관련 부서의 M\&S 관련 발전방향을 참고하고 외주연구 용역과제를 포함한 각종 연구 자료와 대외 M\&S 전문가 및 기관을 통해 육군이 추진해야 할 13 개 과제를 염출하였다. 끝으로 상 기한 목표달성을 위해 세부 추진과제에 대한 M\&S 발전방향을 연구하여 전문가 의견을 수렴하여 수정 보완하여 제시하였다. 이런 연구결과를 기초로 산 - 학 - 연 및 국외 M\&S의 선진 기술에 관한 후속연구가 진행될 필요가 있으며, 다양한 전문가를 통한 M\&S 생태계를 확장하여 육군 분야에 국 한되지 않고, 해군 - 공군의 M\&S 발전방향을 포함한 국방 M\&S 발전을 위한 향후 연구가 진행될 필요성을 제기한다.

\section{Acknowledgements}

We would like to thank Editage (www.editage.co.kr) for English language editing.

\section{Declaration of Conflicting Interests}

The author(s) declared no potential conflicts of interest with respect to the research, authorship, and/or publication of this article. 


\section{Reference}

Bang, S. H., \& Lee, S. L. (2020). A Study on the Linkage Method between Emergency Simulation Model and Other Models. The Journal of the Korea Contents Association, 20(11), 301-313. https://doi.org/10.5392/JKCA.2020.20.11.301

Han, B. G, Kim, Y. H., \& Kang, J. K. (2012). The Vision of ROK Army Training System using M\&S Technology. Journal of the KIMST, 15(5), 667-675. https://doi.org/10.9766/KIMST. 2012.15.5.667

Han, S. H. (2021). A Study on improving Plan of the Evaluating System for Efficient Defense M\&S Accreditation Work. Journal of the Korea Academia-Industrial cooperation Society, 22(4), 42-48. https://doi.org/10.5762/KAIS.2021.22.4.42

Hong, J. H., Sung, C. H., Ahn, J. H. \& Kim, T. G. (2009). Design and Implementation of Interoperable Adaptor for Simulators Interoperation using IEEE 1516 HLA/RTI. Journal of the Korea Institute of Military Science and Technology, 12(1), 88-96. UCI : G704001584.2009.12.1.006

Jeon, J. H., Kim, D. N., \& Lee, S. H. (2020). Analysis of Experience Knowledge from Defense M\&S based on Data Mining Technique: The Case of Defense M\&S for Education and Training. Journal of Korea Technology Innovation Society, 23(6), 1202-1221. https://doi. org/10.35978/jktis.2020.12.23.6.1202

Kim, D. Y., \& Lee, C. G. (2008). Military Defense Modeling and Simulation System Development. Korea Information Processing Society Review, 15(6), 14-24. https://www.koreascience. or.kr/article/JAKO200806942465157.page

Kim, J., Park, S., Cha, J., \& Kim, Y. (2021). Future Tactical Communication System Development Plan. Journal of Convergence for Information Technology, 11(6), 14-23. https://doi.org/10. 22156/CS4SMB.2021.11.06.014

Lee, H. (2020). Trends of developing training in advanced countries and direction of the Army: Focusing on the LVCG training system. Journal of Advances in Military Studies, 3(2), 1-15. https://doi.org/10.37944/jams.v3i2.73

Shin, K., Choi, H., \& Park, S. (2021). Developing a Digital Twin and Extended Reality based Future Integrated Combat Training Platform under 5G. Journal of Digital Contents Society, 22(4), 727-735. https://doi.org/10.9728/dcs.2021.22.4.727

Yoo, S., Kim, J., Lee, S., \& Kim, D. (2020). Defense M\&S development plan according to change 
Future and development ... / Kyoungchan Won • Yong-Pil Kim • Minsuk Kim • Taegyun Ha • Yong-Yeon Lee • Hojun Lee 57

in the defense environment. KIDA Brief, Military-5, 1-4. https://www.kida.re.kr/cmm/ viewBoardImageFile.do?idx=28046 
국문초록

\section{초일류 육군 도약을 위한 21-35 육군 $\mathrm{M} \& \mathrm{~S}$ 발전방향: 첨단과학기술군 육성을 중심으로}

원경찬 ${ }^{*}$ 김용필** - 김민숙** - 하태균 ${ }^{* *}$ - 이용연 ${ }^{* *}$ - 이호준 ${ }^{* * *}$

본 연구에서는 육군비전 2030을 중심으로 첨단과학기술군 육성의 핵심수단인 M\&S의 발전방향을 제시하 였다. 육군은 다양한 도전을 기회로 삼아 '한계를 넘어서는 초일류 육군'으로 도약하고자 한다. 특히, 4 차 산업 혁명에 대비하여 첨단과학기술군으로 거듭나기 위해 노력하고 있다. 육군은 $\mathrm{AI}$ 와 드론봇 전투체계, 워리어플 랫폼, 그리고 보병부대의 기동화 - 지능화 - 네트워크화를 추구하는 'Army-TIGER 4.0' 등을 추진하여 미래전 을 준비하고 있다. M\&S는 육군비전2030을 달성하고 첨단과학기술군으로 도약하기 위한 최선의 솔루션이다. 그 예로 여단급 훈련의 패러다임을 바꾼 과학화전투훈련(KCTC), 전투지휘훈련(BCTP), 헬기 조종시뮬레이터 등 다양한 훈련과 한미연합연습 및 태극연습 등이 M\&S를 통해 이뤄지고 있다. 훈련 분야 이외에도 작전계획 분석, 전시 자원소요분석, 무기체계 소요타당성 분석 등 각종 계획의 신뢰성을 높이고, 군 운영의 효율성을 도 모하며, 새로운 개념과 무기체계의 타당성을 검증하는데도 M\&S를 활용하고 있다. M\&S는 AI와 빅데이터, 가 상현실과 사물인터넷 등의 첨단기술과 융합하여 제한된 예산과 자원 부족의 어려운 여건을 보다 창조적인 사 고와 방법을 통해 극복하는 핵심수단이며 효율적인 국방경영의 필수 요소이다. 육군은 그 중요성을 절감하고 미래 첨단과학기술군으로의 도약적 변혁을 위해 육군 M\&S의 비전과 방향을 설계하고, 세부 실천과제를 지속 적으로 발전시켜 나가고자 21-35 육군 M\&S 발전방향을 제시하고자 한다.

주제어 : 모델링과 시뮬레이션, 첨단과학기술군, 육군비전2030, 과학화훈련

* (제1저자) 육군분석평가단, M\&S 전력계획장교, popdin@naver.com

** (공동저자) 분석평가단장, anwlwow@naver.com; M\&S정책발전장교, ppippo8010@gmail.com; M\&S기반체계장교, positiveha@naver.com; M\&S전력기획장교, c15837@naver.com

*** (교신저자) 육군분석평가단, M\&S 발전과장, jikimy72@naver.com 